\title{
Design, Structural and Electrostatic Analysis of Three MEMS Micro Accelerometers
}

\author{
Jose Luis Gonzalez Vidal $^{\mathrm{a}^{*}}$, Jose Luis. Calderon Osorno ${ }^{\mathrm{b}}$, Edmundo Rene Duran Camarillo ${ }^{\mathrm{c}}$, Silvestre \\ Ascencion Garcia Sanchez ${ }^{\mathrm{d}}$
}

\begin{abstract}
Three low-power MEMS micro-accelerometers were designed, the first with variable distance and the last with variable area. Then, electrostatic analysis and simulation by the finite element method was performed to show its behavior and performance. In the simulation and analysis the software COMSOL MULTIPHYSICS 5.1 was used. The results show that the variable area micro-accelerometer exhibits the best relationship between behavior and performance
\end{abstract}

Index Terms- Inertial sensor, MEMS, mesh, microaccelerometer.

\section{INTRODUCTION}

Accelerometers have a wide application as inertial navigation, inertial guidance systems, riding comfort, among others, that's why accelerometers production is increasing each day. The working principle of accelerometers is the displacement of a suspended mass tied to a frame work, such displacement produces a change of a capacitance due to its own structure; in this case, an accelerometer is a capacitive sensor. On the other hand, as an accelerometer senses change of velocity or movement is considered an inertial sensor [1]$10]$.

The capacitance $\mathrm{C}$ of a parallel plate capacitor is given by:

$$
C=\varepsilon_{0} \frac{A}{d}
$$

Where $\varepsilon_{0}$ is the vacuum permittivity, $\mathrm{A}$ is area of the plates and $\mathrm{d}$ is the distance between themselves (fig. 1).

Micro-accelerometers are a kind of MEMS, generally MEMS can be divided into two categories: MEMS sensors and MEMS actuators. MEMS actuators are based on moving mechanism activated by an electrical signal. Micro sensors are the devices used to detect the change in the environment by measuring its corresponding parameters such as mechanical, chemical, thermal and electromagnetic information [1], [2], [4], [9],[11]-[16].

The goal of this work is to develop tree microaccelerometers MEMS using finite element modelling (FEM), with the aim being to verify their behaviors.[2],[4],[6],[7],[8],[11]-[15] In these cases, microaccelerometers structure design is analysed by mesh, where such mesh separates everything into parts, and each part is

Published Nov 16, 2019

Jose Luis. Gonzalez Vidal ${ }^{\text {** }}$ is with Universidad Autonoma del Estado de Hidalgo, Pachuca, Hidalgo CP 42082 (e-mail: jlvidal@uaeh.edu.mx)

Jose Luis Calderon Osorno ${ }^{b}$ is with Instituto Politecnico Nacional, UPIIH San Agustin Tlaxiaca, Hidalgo CP 42162 Mexico (e-mail: jcalderono@ipn.mx). evaluated one by one [16]-[20]. Electrostatic behavior is when a voltage is applied and structural behaviors occurred with a function step of $70 \mathrm{~g}$. 1

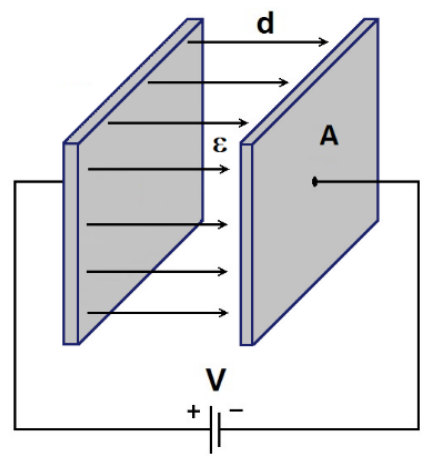

Fig. 1. Parallel plates capacitor.

\section{DESIGN}

The design of the first structure is shown in fig. 2. A central comb (inertial mass) is tied to a couple of rectangular springs at the right and left ends; every tooth of the comb has two teeth electrodes conforming capacitors $\mathrm{C} 1$ and $\mathrm{C} 2$, fig. 3 shows the second structure, in this case inertial mass and electrodes are similar to accelerometer from fig. 2, but end sides of inertial mass are tied to two parabolic anchors. In this case only upper side anchors are joined to the substrate. If both, the upper and under sides are joined to substrate, their rigidity could become higher.

A third structure was designed, in this case, a double fingerboard was utilized. Inertial mass moves on the $\mathrm{X}$ axis, fingers of both upper and under sides conform the capacitors $\mathrm{C} 1$ and C2 (fig. 4), unlike the two previous structures, where the distance between fingers is variable, at this microaccelerometer the area is variable.

\section{Simulation Results AND Discussion}

Once the three accelerometers designed, their behavior was compared. First, a dispersion mesh by finite element analysis was generated for every micro-accelerometer using COMSOL MULTIPHYSICS 5.1 software. Then the deformation, maximum stress and electrostatic dispersion analysis was performed. Finally, analysis of the voltage variation against geometry displacement was performed.

Edmundo Rene Duran Camarillo ${ }^{c}$ is with Instituto Politecnico Nacional, ESCOM, Mexico City CP 07738 Mexico (e-mail: eduranc@ipn.mx).

Silvestre Ascencion Garcia Sanchez ${ }^{\mathrm{d}}$ is with Instituto Politecnico Nacional, UPIIH, San Agustin Tlaxiaca, Hidalgo CP 42162 Mexico (e-mail: sagarcias@ipn.mx). 


\section{A. Rectangular spring accelerometer.}

Figure 5a shows the dispersion mesh of rectangular spring accelerometer, more than 18,000 analysis points were generated. They support the net displacement of the inertial mass that for this case is $1.3 \times 10^{-13} \mathrm{~m}$.

The blue line in fig. 5b, represents the behavior of point 31 , located at the upper end of the internal teeth of the geometry. The behavior of points 62 and 63 are very similar. The behavior of point number 62 is represented by a green line and is located in the central part of the inertial mass, this being where the teeth and the springs are fastened. Finally, the red line represents the behavior of point 63 , which is located in the external teeth of the geometry in the same fig. With this design, it is possible to appreciate that the behavior at points 31 and 63 is very similar, due to the geometry of the rectangular spring.

Figure $5 \mathrm{c}$ shows the moving parts subject to the highest mechanical stress. The electrostatic distribution of the system at a stable state is shown in fig. $5 \mathrm{~d}$, taking into account that the insulating medium used between plates is air at atmospheric pressure and room temperature, where red zone indicates the most positive part and blue zone indicates the most negative part, in the central part it is observed that the electrodes on left side are connected to the positive terminal (5V.), and that the electrodes on the right side are connected to the negative terminal, in this case, at $0 \mathrm{~V}$.

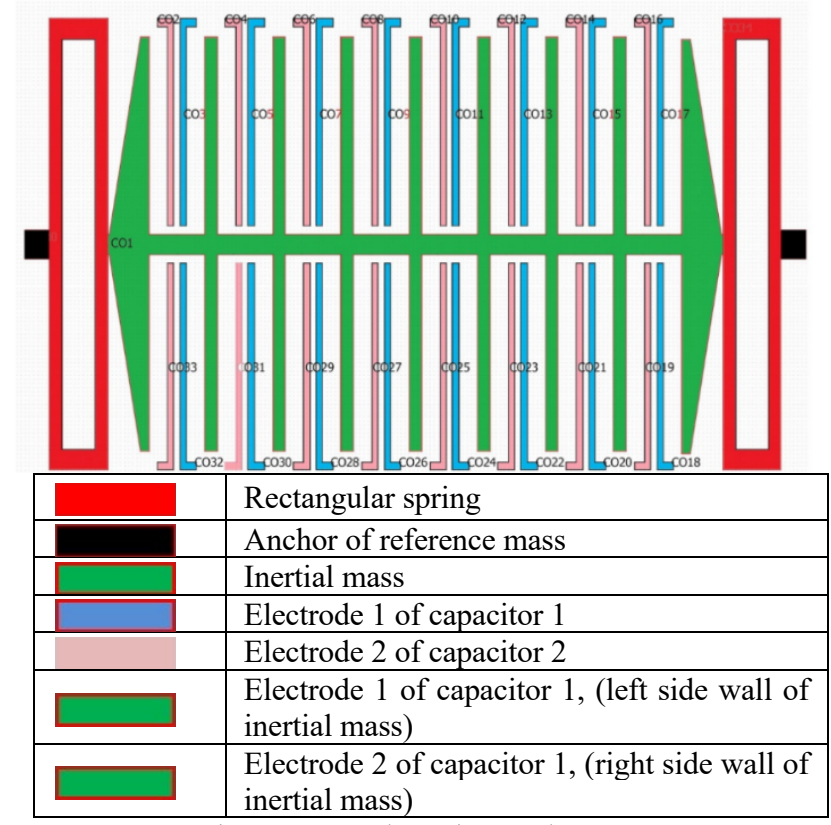

Fig. 2. Rectangular spring accelerometer.

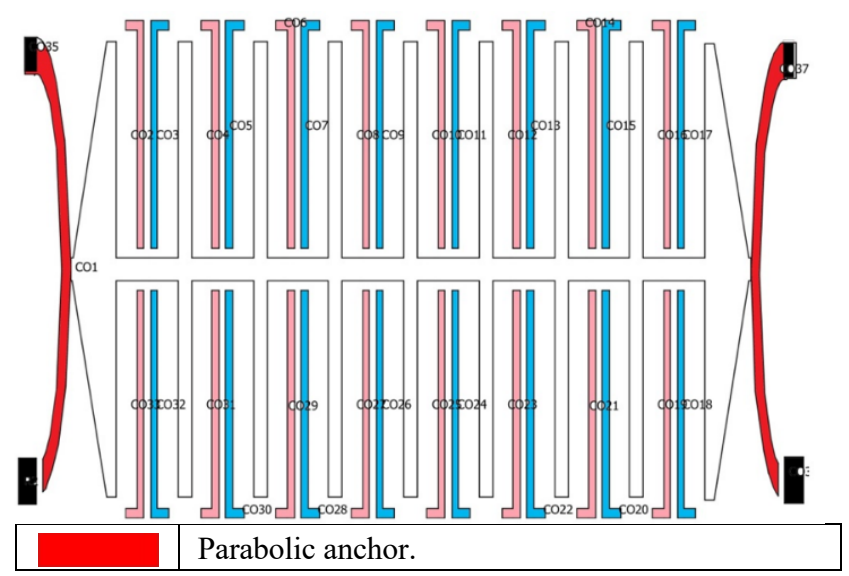

\begin{tabular}{|l|l|}
\hline & Anchor of reference mass. \\
\hline$\square$ & Inertial mass. \\
\hline$\square$ & Electrode 1 of capacitor 1. \\
\hline & Electrode 2 of capacitor 2. \\
\hline$\square$ & $\begin{array}{l}\text { Electrode 1 of capacitor 1, (left side wall of inertial } \\
\text { mass). }\end{array}$ \\
\hline$\square$ & $\begin{array}{l}\text { Electrode } 2 \text { of capacitor 1, (right side wall of } \\
\text { inertial mass). }\end{array}$ \\
\hline
\end{tabular}

Fig. 3. Parabolic spring accelerometer.

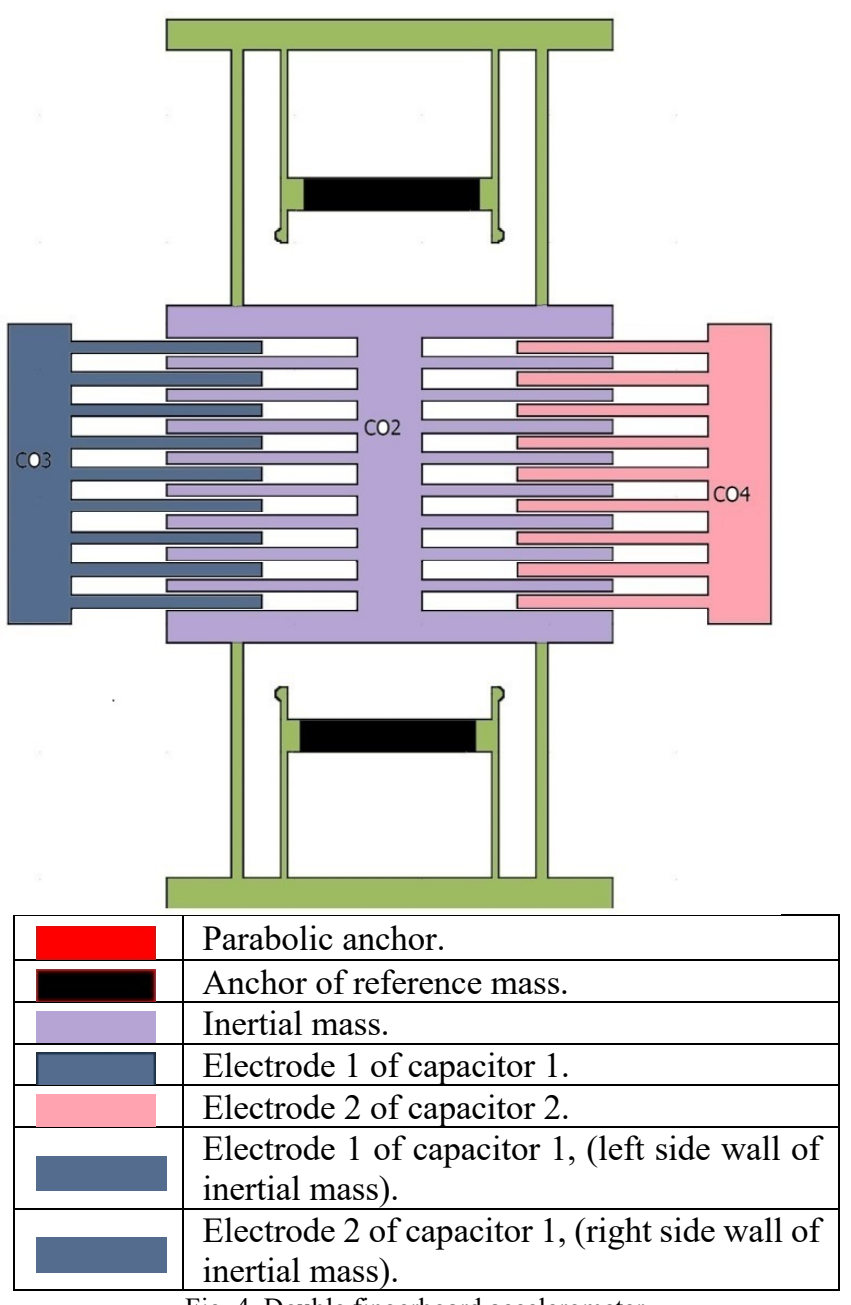

Fig. 4. Double fingerboard accelerometer.

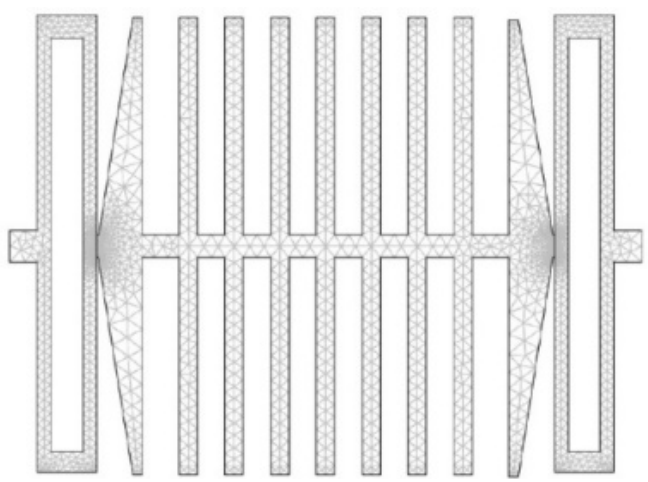

(a) Dispersion mesh of rectangular spring accelerometer by finite elements analysis. 


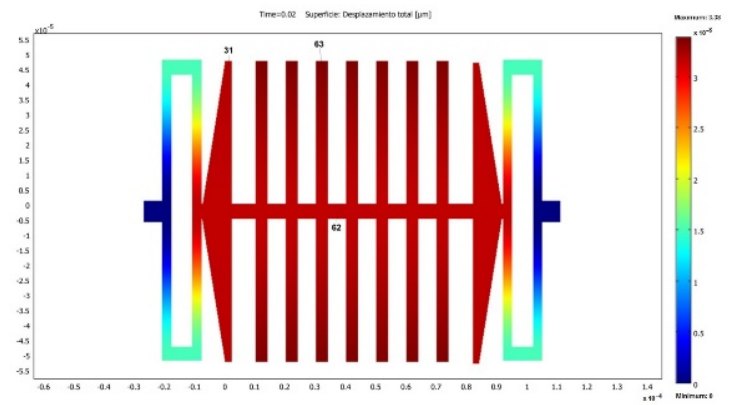

(b) Deformation for an acceleration of $70 \mathrm{~g}$.

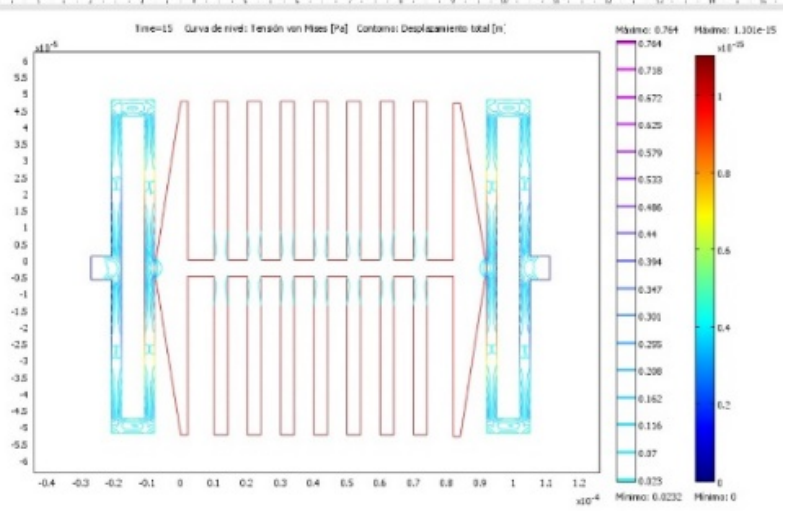

(c) Maximum stress of moving parts.

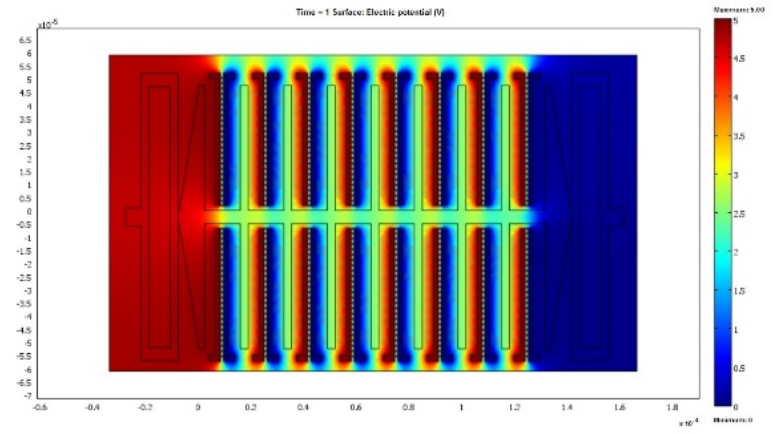

(d) Electrostatic dispersion with inertial mass in stable state.

Fig. 5. Rectangular spring accelerometer analysis.

Figure 6 shows voltage variation of the output electrode against the movement achieved by this geometry is shown, for a voltage range of $2.4022 \mathrm{~V}$ to $2.78 \mathrm{~V}$ the simulated displacement for this geometry ranges from $-2 \times 10^{-13} \mathrm{~m}$ to $2 \times 10^{-13} \mathrm{~m}$. This represents a variation of capacitance. It is more useful because of the signal conditioning and the manipulation of it, and it is less likely to be confused with the environmental noise inherent to the system.

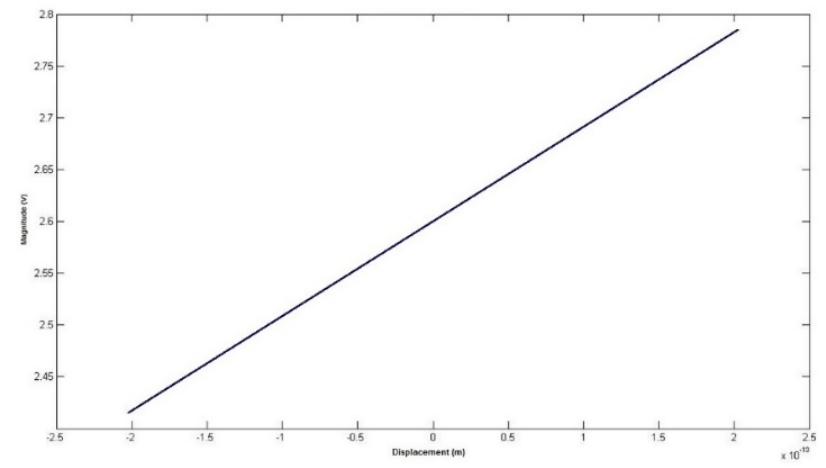

Fig 6. Output voltage plot by displacement of $70 \mathrm{~g}$.

\section{B. Parabolic anchors accelerometer.}

In the case of micro-accelerometer with parabolic anchors, its dispersion mesh for the finite element analysis generated by the COMSOL MULTIPHYSICS 5.1 program is shown in fig. 7a, 25600 elements were obtained.

Figure $7 \mathrm{~b}$ shows micro-accelerometer with parabolic anchors simulation when a $70 \mathrm{~g}$ force is applied; a critical deformation can be noticed, due to the lower parts that are not joined to structure. Additionally, maximum stresses in the inertial mass are seen at the ends of the upper right teeth and at the ends of the lower left teeth, in the central part of the inertial mass medium stresses are appreciated, and in the parabolic springs, the lowest stresses as can be seen in fig. 7c. For the electrostatic dispersion analysis, simulations were performed applying $0 \mathrm{~V}$ to $5 \mathrm{~V}$ to the electrodes of the microaccelerometer, as shown in fig. $7 \mathrm{~d}$. The left region shows a red hue, which indicates the most positive zone, close to $5 \mathrm{~V}$. Furthermore, the right region shows a blue tone, close to $0 \mathrm{~V}$. The right interdigitated fixed teeth show red tones, and the left interdigitated fixed teeth show blue tonalities as well, which indicate the same polarizations.

Figure 8 shows the behavior of the output voltage on the inertial mass. It is not only linear but also the displacement of the mass is greater than in the previous case. This displacement improves its behavior. This non-linearity is attributed to the fact that parabolic springs are only anchored in their upper part. Besides that, the variation of the voltage of the output electrode against the movement achieved by this geometry is shown for a voltage range from $2.0 \mathrm{~V}$ to $2.92 \mathrm{~V}$ approximately. The simulated displacement for this geometry ranges from $-1.5 \times 10^{-6} \mathrm{~m}$ to $1.5 \times 10^{-6} \mathrm{~m}$.

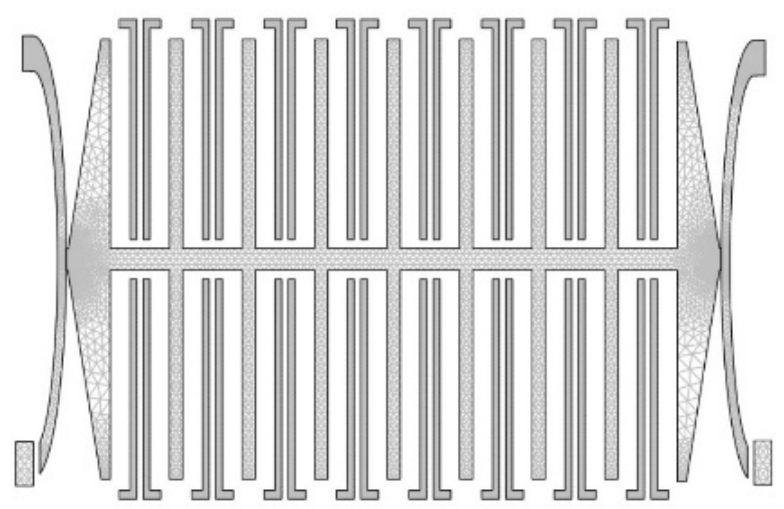

(a) Dispersion mesh for finite elements analysis.

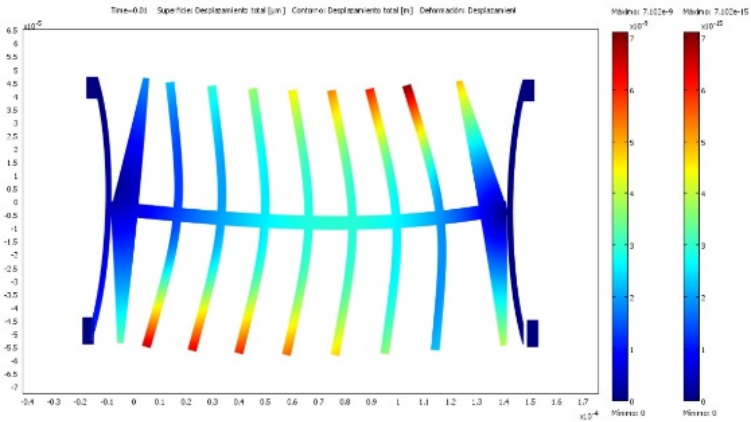

(b) Deformation for an acceleration of $70 \mathrm{~g}$. 


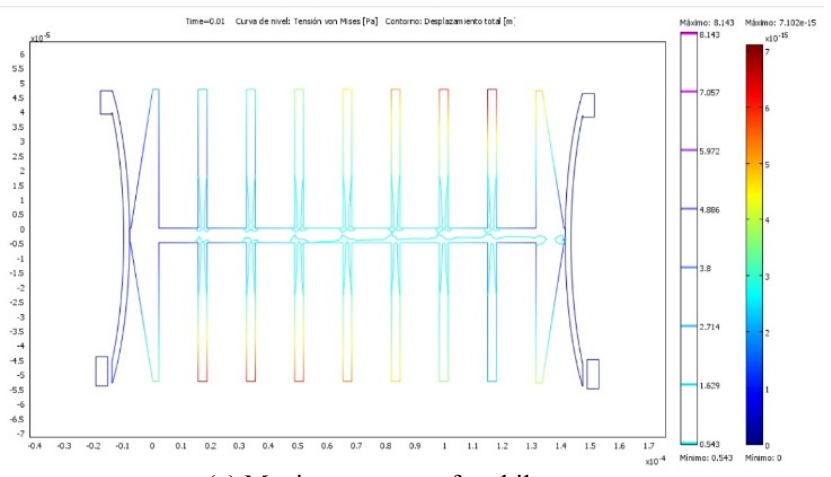

(c) Maximum stress of mobile parts.

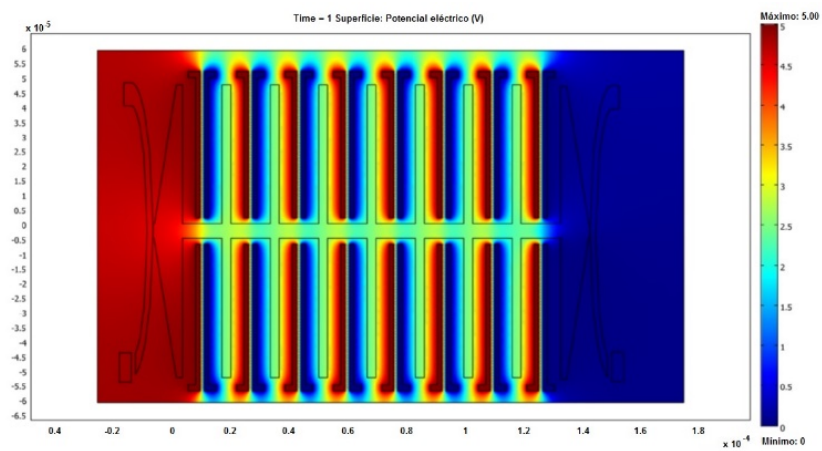

(d) Electrostatic dispersion with inertial mass in stable state Fig. 7. Parabolic anchors accelerometer analysis.

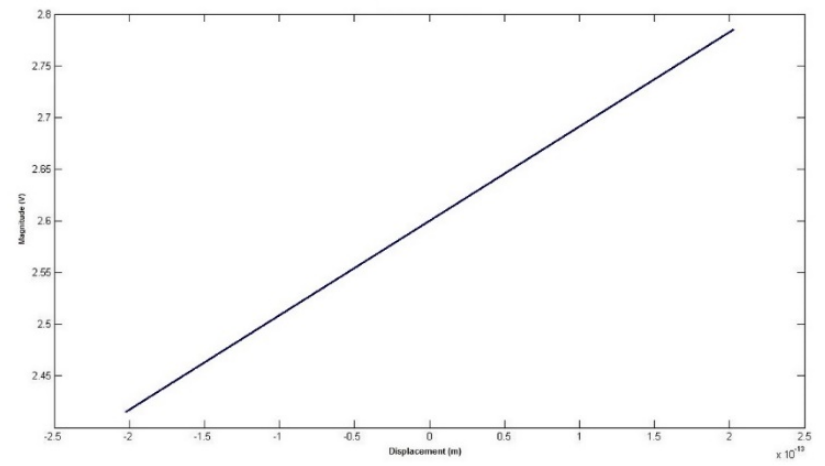

Fig 8. Parabolic micro-accelerometer output voltage plot by displacement of $70 \mathrm{~g}$.

\section{Double fingerboard accelerometer.}

Figure 9a shows the mesh distribution of the double fingerboard micro-accelerometer for the analysis by finite elements by COMSOL MULTIPHYSICS 5.1 program. In addition, in this triangular shaped mesh was developed 14408 points of analysis. On the other hand, the microsystem has been subjected to an acceleration of $70 \mathrm{~g}$, it undergoes a deformation, shown in fig. $9 \mathrm{~b}$, and this deformation produces a change of area between the teeth of the combs and the inertial mass, which produces a change of capacitance in the microstructure. In this case, behavior of both of the double fingerboards is very interesting because the rigidity of the double fingerboard is combined to give greater stability.

Figure 9c shows the maximum stresses in the moving parts of the system, particularly in the adjoining areas of the fixed parts and the moving parts of the double fingerboard and the width. Also the double fingerboard and the inertial mass and the double fingerboard and the mass adhered have a greater stress as well.

The electrostatic dispersion shown in fig. 9d with $5 \mathrm{~V}$ applied to the positive electrode and $0 \mathrm{~V}$ to the negative electrode, can be noticed in the most negative part by the blue located at the left end side of the structure, and in addition in the most positive part in red at the right side of the structure.

Figu10 shows the behavior of the output voltage in the inertial mass. The displacement of the mass is higher than in previous cases and this indicates improvement of its behavior. In this plot, the voltage variation of output electrode against the movement achieved by this geometry is shown, for a voltage range of $1.6 \mathrm{~V}$ to $3.5 \mathrm{~V}$ approximately, the simulated displacement for this geometry ranges from $-5 \times 10^{-}$ ${ }^{6} \mathrm{~m}$ to $5 \times 10^{-6} \mathrm{~m}$. This voltage variation is attributed to the distance between the teeth is $1.5 \times 10^{-6} \mathrm{~m}$

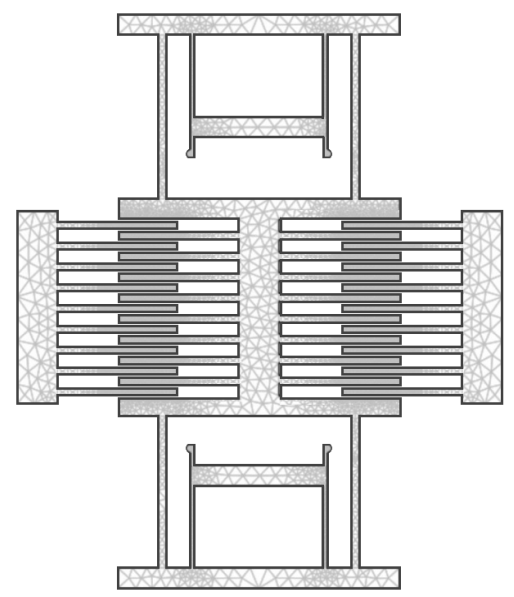

(a) Dispersion mesh for finite elements analysis of double fingerboard

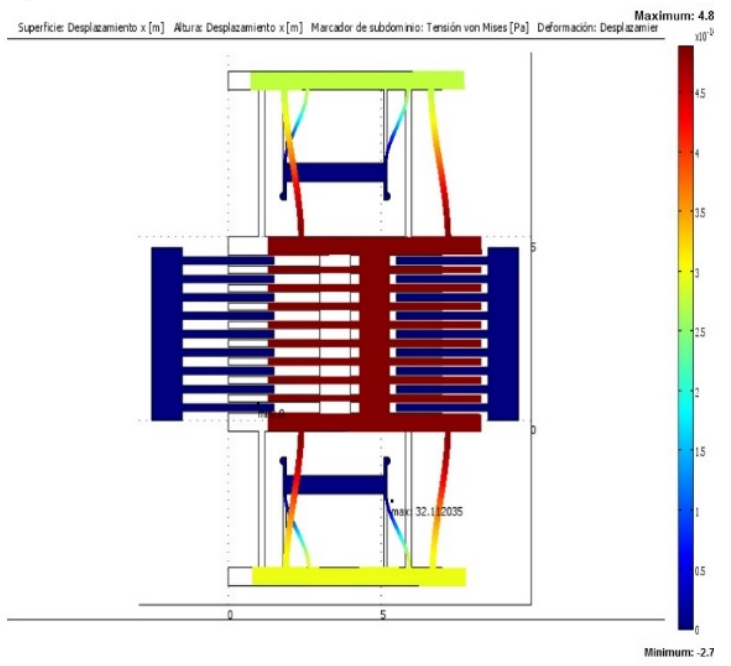

(b) Deformation for an acceleration of $70 \mathrm{~g}$.

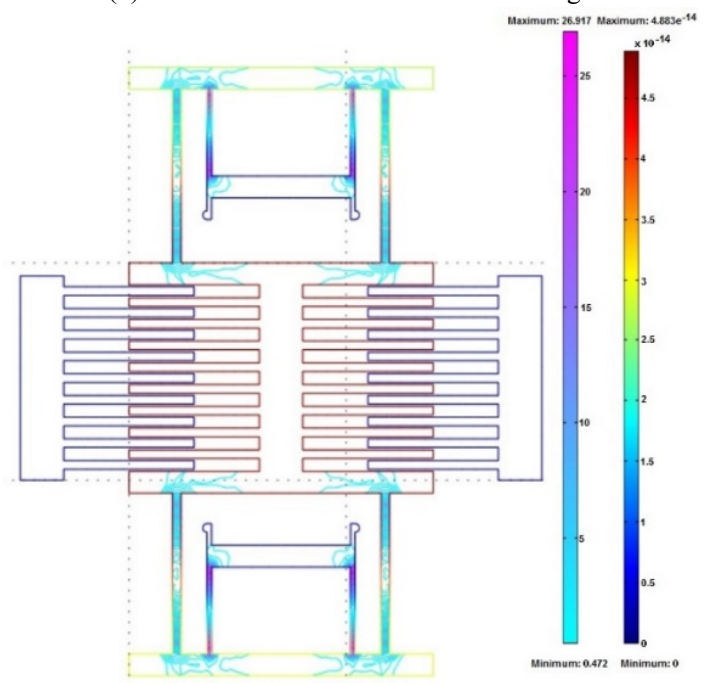

(c) Maximum stress of mobile parts. 


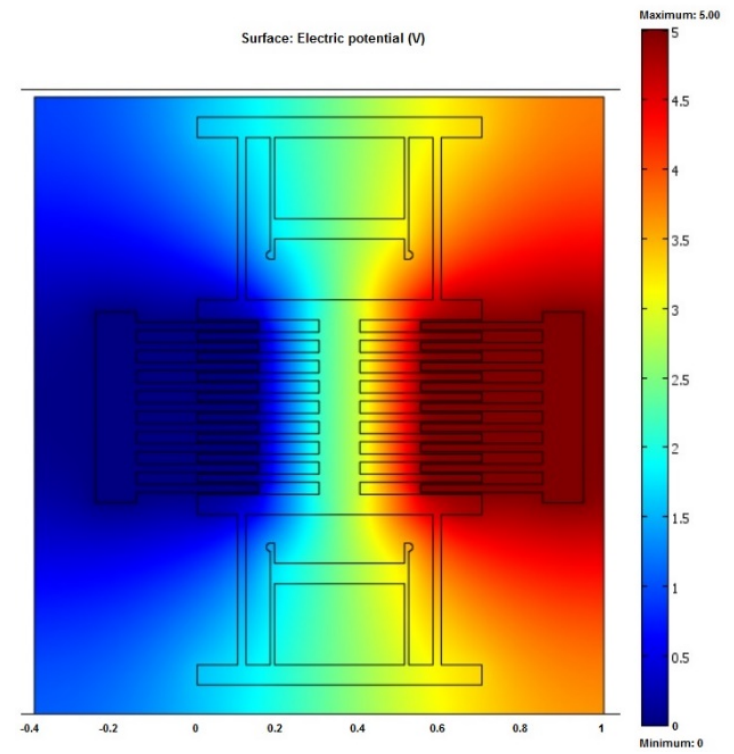

(d) Electrostatic dispersion with inertial mass in stable state. Fig. 9. Double fingerboard accelerometer analysis.

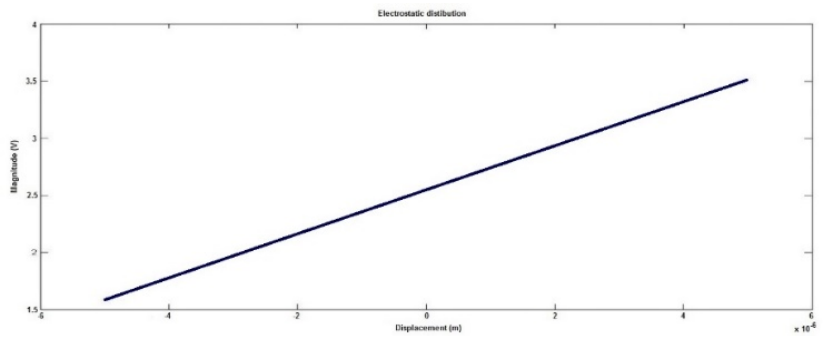

Fig 10. Double fingerboard micro-accelerometer output voltage plot by displacement of $70 \mathrm{~g}$.

\section{CONCLUSIONS}

The properties from electrical point of view of the MEMS were studied, as well as their application in capacitive microsensors. With capacitive sensors not only the change of distance between plates, but also change of area between plates was studied. In addition, mechanical properties of the spring mass system were studied, in this case microaccelerometers MEMS.

Three designs were made and simulated, two microaccelerometers with variable distance. Rectangular spring micro-accelerometer. This design is the most commercial. In the ADX series of Analog Devices, its equations are not very complicated. They present greater displacement of the inertial mass and therefore, greater sensitivity. Parabolic spring micro-accelerometers, this design is proposed as a new alternative, no similar designs have been registered in the literature, its main feature is that it generates a greater range of movement of the inertial mass. Its drawback is that it presents an asymmetric behavior at the ends of the teeth of the inertial mass.

For the case of variable area micro-accelerometer, the double fingerboard micro-accelerometer with change of area design is the most complex due to its structure, but it is the one that presents better behavior. The change of area between the teeth of the electrodes and the inertial mass allows greater displacement which it is reflected in greater sensitivity, in addition to a linear behavior in the variation of the capacitance.
For the characterization of these microstructures, multiple simulations were performed with the COMSOL MULTIPHYSICS 5.1 software.

\section{ACKNOWLEDGEMENT}

The authors thank to the Superior Computer School of the Instituto Politecnico Nacional for the support received and the granted facilities for the development of this project. This article is derived from research projects with registration. SIP 20181880, 20195552 and 20190095

\section{REFERENCES}

[1] S. Beeby, G. Ensell, M. Kraft and N. White, MEMS Mechanical Sensors, Norwood, MA: Artech House, Inc., 2004.

[2] D. Hernández, Desarrollo de Prototipos para Acelerometros Mems, Mineral de la Reforma., Hidalgo, UAEH, 2009.

[3] G. K.Fedder, S. Santhanam, M.L.Reed, S. C. Eagle, D. F. Guillou and M. S.-C.LuaL.R.Carleya, "Laminated high-aspect-ratio microstructures in a conventional CMOS process," Sensors and Actuators A: Physical, vol. 57, no. 2, pp. 103-110, 1996.

[4] C. R. Marra, F. M. Ferrari, G. Langfelder, A. Tocchio and F. Rizzini, "Single resonator, time-switched, low offset drift z-axis FM MEMS accelerometer," in Inertial Sensors and Systems (INERTIAL), 2018 IEEE International Symposium on, Lake Como, Italy, 2018.

[5] N. Maluf and K. Williams, An Introduction to Microelectromechanical Systems Engineering, Second Edition, 2nd ed., Norwood, MA: Artech House Inc., 2004.

[6] A. S. Khan and T.Shanmuganantham, "Arc-Shaped Cantilever Beam RF MEMS Switch for Low Actuation Voltage," Proceedings of 2017 IEEE International Conference on Circuits and Systems (ICCS 2017), 2017.

[7] I. Nevludov, V. Yevsieiev, V. Bortnikova and S. Miliutina, "MEMS Accelerometers Production Technological Route Selection," in the Experience of Designing and Application of CAD Systems in Microelectronics (CADSM), 2017 14th International Conference The, Lviv, Ukraine, 2017.

[8] M. A. Shahbaz, Z. H. Warsi, S. M. Irshad, S. T. Irshad and S. A. Jawed, "Design and Analysis of CMOS MEMS Based Single Proof Mass Tri-axial Capacitive Accelerometer with Readout Integrated Circuit," in Electrical Engineering and Computing Technologies (INTELLECT), Karachi, Pakistan, 2017.

[9] K. J. V. S. G. Vijay K. Varadan, Smart Material Systems and MEMS: Design and Development Methodologies, Chichester: Wiley \& Sons Ltd, 2006.

[10] D. Analog, "http://www.analog.com," Analog Devices, mayo 2018. [Online]. Available: http://www.analog.com/en/products/sensorsmems/accelerometers.html. [Accessed Mayo 2018].

[11] D. Rana and M. Kaur, "Design and Simulation of CMOS MEMS Accelerometer Behavioral Model," in Wireless Networks and Embedded Systems (WECON), Rajpura, India, 2016.

[12] R. M. Kittali, B. G. Sheeparamatti and A. Sheeparamatti, "Modeling and analysis of MEMS based Accelerometer in Simulink," in Smart Technologies For Smart Nation, 2017 International Conference On, Bengaluru, India, 2017.

[13] A. Kuncar, M. Sysel and T. Urbanek, "Calibration of Low-Cost Accelerometer and Magnetometer with Differential Evolution," in 2017 International Conference on Military Technologies (ICMT, Brno, Czech Republic, 2017.

[14] A. Basuwaqi, M. H. M. khir, A. A. Y. Ahmed, A. S. Rabih, M. U. Mian and J. O. Dennis, "Effects of Frequency and Voltage on the Output of CMOS-MEMS Device," in 2017 IEEE Asia Pacific Conference on Postgraduate Research in Microelectronics and Electronics (PrimeAsia), Kuala Lumpur, Malaysia, 2017.

[15] X. Lui, W. Jiang, L. Zhao, C. Jia, M. Yu and Z. Jiang, "Liquid packaging effects on piezoresistive MEMS Accelerometer," in Inertial Sensors and Systems (INERTIAL), 2018 IEEE International Symposium on, Lake Como, Italy, Italy, 2018. 
[16] J. L. Gonzalez-Vidal, “Aplicación de estructuras micro-electromecánicas (MEM's) con tecnología s, para sensores de parámetros físicos," Ph. D. dissertation, CINVESTAV-IPN, México, 2006.

[17] Dhineshkaarthi.K, S. L. Preeth and R. Kumar, "MEMS Cantilever based identification of Carcinogenic MZN," in 2017 International Conference on Electrical, Instrumentation and Communication Engineering (ICEICE2017), Karur, India, 2017.

[18] Y. Zhang, Y. Yu, Z. Zhang and X. Zhang, "Structure and Design of Microgrippers: A Survey," in Cybernetics, Robotics and Control (CRC), 2017 2nd International Conference on, Chengdu, China, 2017

[19] W. Wu, Z. Li, J. Liu, J. Fan and L. Tu, "A NANO-G MEMS ACCELEROMETER FOR EARTHQUAKE MONITORING," Transducers 2017, Kaohsiung, TAIWAN, June 18-22, 2017, pp. 599-602, 2017.

[20] C.-L. Lu and M.-K. Yeh, "Thermal stress analysis of chip with pressure sensor embedded in accelerometer," in Electronics Packaging (ICEP), 2017 International Conference on, Yamagata, Japan, 2017.

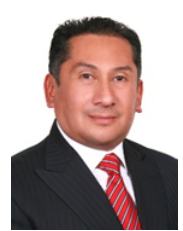

Jose Luis González Vidal. Obtained the title of electronics enginner from Instituto Tecnologico of Orizaba in Orizaba, Mexico in 1991. Obtained a master's degree and Ph.D by the CINVESTAV of the Instituto Politecnico Nacional in Mexico city in 1994 and 2006, respectively.

$\mathrm{He}$ was a PROFESSOR at Instituto Politecnico Nacional and the Instituto Tecnologico de Estudios Superiores de Monterrey. $\mathrm{He}$ is currently the LEADER OF THE MICROELECTRONICS LABORATORY of the Institute of Basic Sciences and Engineering, Universidad Autonoma del Estado de Hidalgo in Pachuca, Mexico. His research is related to the design and testing of electronic systems, large-scale integrated circuits and mixed and analog signal MEMS.

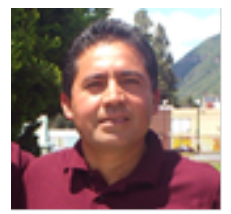

Jose Luis Calderon Osorno. Obtained the title of electronics engineer from Instituto Tecnologico de Orizaba in Orizaba, Mexico in 1991. Obtained a master of science degree from the CINVESTAV in the Instituto Politecnico Nacional in Mexico City, Mexico in 1995 .

He was a PROFESSOR of the Escuela Superior de Computo in the Instituto Politecnico Nacional from 1995 to 2015. Currently, he is a FULL PROFESSOR in the Unidad Profesional Interdisciplinaria de Ingenieria Campus Hidalgo, Instituto Politecnico Nacional in San Agustin Tlaxiaca Mexico. His areas of interest are: artificial intelligence, neural networks, genetic algorithms, expert systems, fuzzy control systems, artificial vision, pattern recognition and robotics.

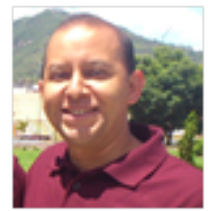

Edmundo Rene Duran Camarillo. Obtained the title of Electronics Engineer from Instituto Tecnologico de Orizaba in Orizaba, Mexico in 1993. Obtained the master of science degree from the CINVESTAV, Instituto Politecnico Nacional in Mexico city, Mexico in 1994.

He is a SENIOR RESEARCH PROFESSOR at the Escuela Superior de Computo, Instituto Politecnico Nacional in Mexico City, Mexico. His areas of interest are: automation and control, artificial intelligence, neuro-fuzzy systems, artificial neural networks, and expert systems.

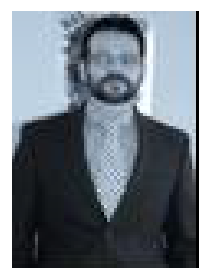

Silvestre Ascencion Garcia Sanchez. Born in Madero city, Mexico in 11 July of 1975 . Obtained the title of physics from Universidad Nacional Autonoma de Mexico in Mexico City, Mexico in 2003. Obtained a master of science degree from the Escuela Superior de Ingenieria Mecanica y Electrica, Campus Culhuacan, Instituto Politecnico Nacional in Mexico City, Mexico in 2003.

He was FULL PROFESSOR at ESIME CULHUACAN, Instituto Politecnico Nacional from 1999-2016 and ASOCCIATE GRADUATE PROFESSOR at SEPI ESIME CULHUACAN, Instituto Politecnico Nacional from 2005-2008. Actually, he is a FULL PROFESSOR in the Unidad Profesional Interdisciplinaria de Ingenieria Campus Hidalgo, Instituto Politecnico Nacional in San Agustin Tlaxiaca Mexico. His areas of interest are: artificial intelligence, neural networks, genetic algorithms, data mining and adaptive filters, 